\title{
Cluster-based approach for successful solving real-world vehicle routing problems
}

\author{
Emir Žunić \\ Info Studio d.o.o. Sarajevo \\ and Faculty of Electrical \\ Engineering, University of \\ Sarajevo, B\&H \\ emir.zunic@infostudio.ba
}

\author{
Dženana Đonko \\ Faculty of Electrical \\ Engineering, University of \\ Sarajevo, Bosnia and \\ Herzegovina \\ ddonko@etf.unsa.ba
}

\author{
Haris Šupić \\ Faculty of Electrical \\ Engineering, University of \\ Sarajevo, Bosnia and \\ Herzegovina \\ hsupic@etf.unsa.ba
}

\author{
Sead Delalić \\ Faculty of Science, \\ University of Sarajevo and \\ Info Studio d.o.o. Sarajevo, \\ $\mathrm{B} \& \mathrm{H}$ \\ delalic.sead@pmf.unsa.ba
}

\begin{abstract}
Vehicle routing problem as the generalization of the Travelling Salesman Problem (TSP) is one of the most studied optimization problems. Industry itself pays special attention to this problem, since transportation is one of the most crucial segments in supplying goods. This paper presents an innovative cluster-based approach for the successful solving of real-world vehicle routing problems that can involve extremely complex VRP problems with many customers needing to be served. The validation of the entire approach was based on the real data of a distribution company, with transport savings being in a range of $10-20 \%$. At the same time, the transportation routes are completely feasible, satisfying all the realistic constraints and conditions.
\end{abstract}

Keywords-Clustering, Transport, Vehicle Routing Problem, Feasible solutions, Multi-phase approach

\section{INTRODUCTION}

$\mathrm{V}$ EHICLE routing problem (VRP) is the generalization of the problem of the commercial traveler, and is basically the process of selecting the set of the most appropriate roads and routes for the vehicles from the available fleet of vehicles during the serving of the set of customers (delivery points), resulting in the lowest total cost of delivery of goods and properties. The vehicle routing problem is one of the most studied problems in the scientific and academic community, with constant attempts of implementing more powerful and advanced approaches for solving this problem. In addition, the industry itself pays a lot of attention to this problem, and the reason for this is the potential reduction of the delivery costs and the possibility of the significant financial savings in the process. The central problem in the logistics of one company is the optimization of transport and the management of the transport fleet of vehicles in an optimal way. If the solution of this problem is left to the computer, there are multiple benefits: the obtained solution is optimal, more efficient and financially favorable, and at the same time spends less time and resources on planning and organizing the transport of goods. However, nowadays, many companies use experimental methods more often during the optimization of the transport of goods compared to some modern approaches and algorithms, and the main reason is that there are very few practically applicable solutions that are able to successfully solve the complex VRP problems, which can often be non-standard, while satisfying all the realistic constraints.

On the other hand, with the progress of logistics processes in the early 1950s [1], there has been a lot of research focused on their various applications. With the globalization of this process, the importance of logistics management has significantly grown in the last few years. Logistics tries to optimize existing distribution processes. One of the most important elements in the logistics chain is the transportation system. According to numerous studies, transportation presents one third of the total logistics cost, and transportation systems significantly affect the performances of the complete logistics system. Transportation is necessary in the complete process of production of goods, from the very production to delivery of goods to the final customers. It is possible to get the maximum benefit for distributors and manufacturers only with good coordination among all the components. Logistics planning cannot reach its full potential without a well developed transportation system. Therefore, good transportation systems can increase the efficiency, reduce the operating costs and increase the service quality. The success of solving VRP problems can significantly improve processes in the transportation part of business of each company. Variants of VRP problems differ according to the number of depots (one or more of them), maximum allowed duration or the length of the vehicle route, different vehicle capacities, customers' requests for delivery or collection of certain amounts of goods during service and time windows within which it is necessary to start and finish customer service, as well as time windows (working hours) of vehicles, illustrated in Fig. 1. In a realistic environment, it is important to take into consideration a great number of additional constraints, usually being the result of the specific loading locations and/ or unloading, specific business processes of the distribution company or legal decisions and obligations (e.g. compulsory rest breaks for drivers). These problems are called Rich Vehicle Routing Problems RVRP.

This work was supported by Info Studio d.o.o. Sarajevo 


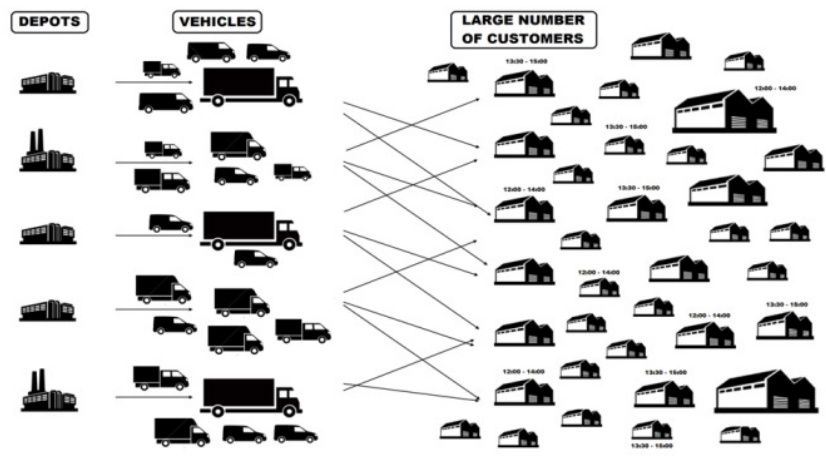

Fig. 1 Vehicle Routing Problem

Transport managers with long-term experience consider an idea to create the transport routes in complex realistic situations primarily from independent clusters joined by the vehicles from the available fleet. Transport routes are obtained by most distribution companies in this way. Most of the available software solutions trying to successfully solve this problem with distribution companies work on the same principle of grouping customers into the regions (clusters), and it is possible to connect several neighboring regions into the same route. This approach usually gives feasible transport routes which is the most important fact from the aspect of the practical application of such an algorithm. For that reason, the obtained routes are very logical and understandable, easily feasible and meet the needs of any of the companies that offer the transportation of goods in their activities, with the special emphasis on logistics. The motivation for the development of the cluster-based algorithm was precisely this: being able to solve the VRP problem while accepting the given real constraints and conditions.

The literature used for this work is presented in Section 2, with the emphasis on the most powerful algorithms for successfully solving real VRP problems. Section 3 presents the algorithm for successfully solving VRP problems based on the clusters (regions), while the obtained results on the realistic set of instances used for the validation can be seen in the Section 4. Conclusions of the work, as well as the guidelines for future research in this area are mentioned in Section 5

\section{RELATED WORK}

During the analysis of the available literature, systems made for application in real environments were considered very interesting. These problems were mainly solved with heterogenic fleets of vehicles, also belong into the HF problems. OR/MS Today, published in June 2006, contains an overview of 17 advanced commercial software solutions capable of solving the instances for more than 1000 locations (customers), and creating 50 and more routes in less than two hours [2]. For the practical application, the vehicle routing is one of the most difficult problems of operational research. For example, every day more than 100.000 UPS (United
Parcel Service, global mail delivery company) drivers follow the computer - generated routes and deliver on average 15.6 million packages.

VRP problems with the included restrictions are of a great importance. The most outstanding constraint in the vehicle routing process are time windows. The time window represents the time interval during the day when it is allowed to visit the customer and deliver him the articles. In Work [3], the authors present the mathematical model for the vehicle routing problem with time windows (VRPTW), a version of the VRP problem suitable for delivery planning in towns with delivery time restrictions. However, many towns in the world limit the delivery time for central areas in the morning hours. A model with the exact solution for a smaller number of instances is used in this work and it compares the performances with the larger instances on a modified genetic algorithm, as well as the process of Taboo search. The results do not show the advantage of any of the algorithms, but confirm that the delivery cost has been significantly increased by introducing these conditions. The modified memetic algorithm for finding the minimal distance in vehicle routing problem with the time windows is presented in work [4]. Various metaheuristic approaches for solving VRPTW problems have been described in work [5], such as the Particle Swarm Optimization (PSO), Ant Colony Optimization (ACO) algorithm and Artificial Bee Colony (ABC) algorithm. A hybrid algorithm combining ACO and the Firefly Algorithm (FA) algorithm was presented in work [6]. The VRPTW problem was observed in detail as a part of this paper. An advanced simulated quenching algorithm was described and applied to VRPTW problem in work [7].

Nowadays, the vehicle routing problem is very important in the scientific community, as it is stated in work [8]. This work describes the research status of this scientific field, including different variants of vehicle routing problem. This paper focuses on the application of nature - inspired algorithms and the most popular classical approaches. Implementations of many algorithms have been described and compared: Genetic algorithm (GA), Taboo search, Simulated quenching (SA), Particle Swarm Optimization (PSO), Bee Colony Optimization (ABC), ant Colony optimization (ACO), Ant Colony Optimization (ACO), Cuckoo Search, Imperialist Competitive Algorithm (ICA), Bat Algorithm (BA) and Firefly Algorithm (FA). Each of these algorithms includes several parameters with their settings determining the result and the quality of the obtained routes.

Several papers from the available literature dealing with the practical realization of VRP problems basically use an algorithm and a principle described in detail and suggested in work [1]. In this work, the authors include the mathematical formulation of the problem, which is easy to understand and can be adapted to the practical problem, and the very way of finding the optimal routes is quite intuitive. The authors propose the clustering (grouping customers into 
the clusters) to be included in the first place. Clustering is based on the geographical characteristics of the customer, time constraints, and the constraints in the form of quantity of goods to be delivered. This work was the starting point for the approach presented later in this paper, with significant improvements in both performance and algorithm terms. It was also used as the integral part of the algorithms proposed in works [10]-[16]. All these facts indicate that the clusterbased approach is exceptionally suitable for solving such problems, both as an individual algorithm, as well as the starting point of the more complex multiphase algorithms.

\section{Proposed Cluster-Based ApProach}

The key element in the supply chain is the transportation system which unites different activities, spatially and temporally divided. Transport includes one third of the logistic costs, and significantly affects the performances of the logistic system. Distribution companies are usually not able to optimize their transportation activities in the most efficient way, and thus lose significant financial resources. Complex VRP problems are divided into mega-clusters (regions) in their initial phase being geographically remote from the depot. In this way, division into logical geographic regions is primarily done, with the best solution being found there; in fact, the vehicles are assigned for the purpose of serving each of the regions, respecting all the constraints.

The first phase in the entire approach is to make megaclusters for all the customers needed to be served. Mega clusters are formed depending on the distance of their centre from the depot. The initial creation of the mega clusters can be attained by using the simplest clustering methods, such as $k$-Means, $k$-Medoids or hierarchical techniques of clustering. In this way, the set of several hundred (or even thousands) customers can be divided into several remote clusters, which significantly reduce the complexity of the problem in the next phases of the proposed approach.

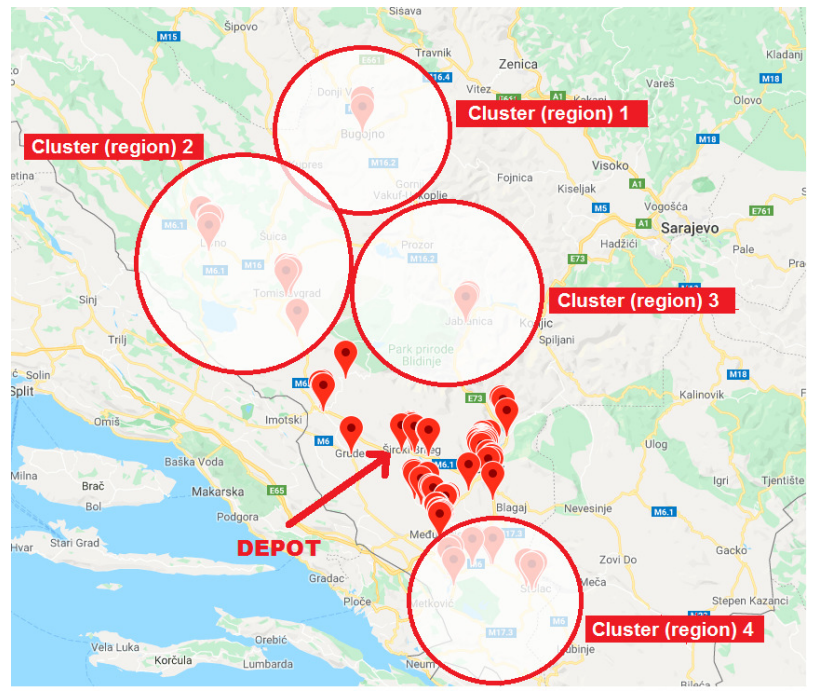

Fig. 2 Customer division in geographically divided regions (clusters)
In the example shown in Fig. 2., special vehicles from the available vehicle fleet during the creation of the transport routes are used for each of the individual clusters and they cannot be combined with each other. Customers who do not belong to any of the clusters are optimized by using the remaining vehicles in the next phase of the approach, and can be combined with each other into one or more transport routes. Usually, the customers around the depot are without any clusters, while the remote customers belong to specific clusters, which is usually the approach being the most acceptable in practice. If there are not enough customers to fulfil the most efficient vehicle in the specific cluster, customers who do not belong to any of the clusters and are in the right direction, can be joined, too.

The number of clusters is determined in the way that within each cluster there has to be minimum: (Number of customers of the cluster / Total number of customers $) \geq[(1 /$ Number of remaining vehicles in the fleet)] $\cdot 100 \%$. The number of clusters described in this way should not exceed the number of available vehicles in the fleet. If this is the case, the smaller, usually neighboring clusters are merged into bigger ones (regions). If there is not any possibility of cluster merging due to the ordered capacity of goods compared to the dimensions and limitations of the available vehicles, then additional (fictive) vehicles are introduced, with a significantly higher delivery cost. Every company can rent additional vehicles from outside companies dealing the delivery of goods as their primary activity.

In order to clarify the proposed approach in mathematical terms, the routing network is being considered and presented by the directed graph $G\{I, P, A\}$ which connects the nodes presenting the customers $I= \begin{cases}i_{1}, i_{2}, \ldots, i_{n} \\ \text { and the nodes }\end{cases}$ presenting the depot through the set of directed branches $A=\{(i, j) \mid(i, j) \in(I \cup P)\}$ It is assumed that the branch $(i, j) \in A$ is the route with the lowest cost which merges the nodes $i$ and $j$. At the location of each customer $i \in I$, a fixed load should be delivered $w_{i}, v l_{i}$ within the time window $\left[a_{i}, b_{i}\right]$, where $a_{i}$ is the earliest time serving the customers should start, and $b_{i}$ is the latest time serving should finish.

A fleet of heterogeneous vehicles $V=\left\{v_{1}, v_{2}, \ldots, v_{m}\right\}$ with different cargo capacities $\left(q_{v}, q_{v}{ }^{V}\right)$, located in several depots, $p \in P$ is used for delivery. Each vehicle $v$ has to leave the assigned depot $p \in P$, deliver the goods to several destinations and come back into the same depot $p$. Thus, vehicle route $v$ is the route consisting of knots $r=(p, \ldots, i, i+1, \ldots, p)$ connected by directional branches belonging to set $A$, and each route starts and finishes in depot $p$ assigned to the vehicle $v$. A pair of matrices depending on the vehicles $C=\left\{c_{i j}{ }^{\nu}\right\}$ and $\Gamma=\left\{t_{i j}{ }^{\nu}\right\}$ is assigned to the set of branches $a_{i j} \in A$. They indicate the cost and the time of the travel from the node $i$ into the node $j$ using the vehicle $v$, respectively. It is assumed that the inequality of the triangle is satisfied by the elements $c_{i j}$ and $t_{i j}$, actually it is: $c_{i k}+c_{k j} \geq c_{i j}$ and $t_{i k}+t_{k j} \geq$ $t_{i j}$. The total required mass and the volume of the goods 
$\left(w_{i}, v o l_{i}\right)$, as well as the serving time by the vehicle $v\left(s t_{i}{ }^{\nu}\right)$ are given for the node $i$.

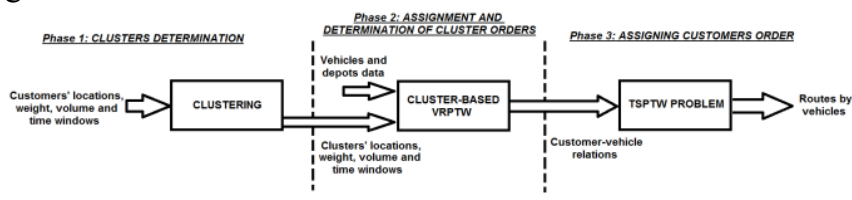

Fig. 3 Phases and the components of the hierarchical hybrid approach

The solution of this problem has to fulfill the following constraints: (i) each route must start and end in the same depot; (ii) each node must be serviced by one and only one vehicle; (iii) the node must not be served by a vehicle with the constraint that it cannot visit that node; (iv) the total load assigned to vehicle $v$ must never exceed its capacity $q_{v}$ and $q_{v}{ }^{V} ;(\mathrm{v})$ the length of time vehicle $v$ is active should be less than the maximum allowed length of working time $t v_{v}{ }^{\max }$; (vi) the customer $i$ service must be completed within the interval $\left[a_{i}, b_{i}\right]$, otherwise penalties will have to be paid. The main aim of the problem is to minimize the total cost of servicing for all the customers. There are four types of costs being considered to fulfill the aim: fixed costs for used vehicles, distance-related costs, costs related to the travelling time along the selected routes, costs related to the waiting time, penalty costs for disrupting time windows and total working hours.

The previously mentioned algorithm [9], which was originally improved, modified and tested over the input data set, combines the clustering methods into the optimization framework, illustrated in Fig. 3.

The algorithm is based on the traditional first cluster then route philosophy. Node clusters are firstly defined; then, such clusters are assigned to the vehicles and put in order on the assigned routes, while the routing and the scheduling are made particularly for each of the individual route in terms of the original nodes. A three-phase VRPTW hierarchical hybrid approach is defined in this phase of the total proposed approach.

Finding a good cluster set, with each of the clusters consisting of several customers, without having any information about the routes is a very difficult assignment. Therefore, a heuristic algorithm based on time windows is used for efficiently arranging the customers into a small number of the possible clusters. This clustering procedure leads to the compact version of VRPTW formulation presented below, by replacing the nodes with the clusters. The clustering procedure and the compact VRPTW model are basic building blocks of the proposed hybrid approach. After grouping the customers into the clusters during the Phase 1, the VRPTW problem of a small dimension is being solved and formulated in terms of clusters rather than nodes. This solution enables clusters to be assigned to the vehicles and to construct the routes by collecting the clusters into the same route (Phase 2). The detailed routing for each of the routes found in Phase 2 is made in the last phase. In Phase 3, the number of routing problems are solved for the number of visitors that have to visit the routes. The aim is to solve the more compact form of the basic VRPTW formulation. The model takes into consideration only the nodes within the cluster of the route being analyzed. As the order of visiting the clusters has already been determined in Phase 2, the relative order among the nodes belonging to different clusters is already familiar, as well as the values of numerous variables $S_{i j}$. This causes a significant reduction of the number of binary variables and constraints in the model solved in Phase 3.

i) Heuristic clustering algorithm (Phase 1)

The aim of Phase 1 is to significantly decrease the calculations for the later phases. This aim is accomplished by defining the small set of feasible clusters or "hyper nodes", where each of them include several customers, and then by establishing the approximate distances and travel time between any of the two clusters. As the mathematical model contains several clusters, and not a large number of customers, dimensionality of the VRPTW problem can be significantly decreased. In order to find a good set of clusters for big problems, the following heuristic procedure is used:

1) a) Open the list of nodes $L$, and sort them according to the ascending order of time $a_{i}$. If some of the nodes have the same $a_{i}$, sort them in ascending order of time $b_{i}$.

b) Open the list of the available vehicles $V$, and sort them according to the descending order $\left(q_{v} / c f_{v}\right)$.

c) Select the maximum allowable distance between any of the two nodes within the same cluster $\left(d^{\max }\right)$ and the maximum allowable waiting time $\Delta$.

2) ( $n$-th main iteration) Open the empty list $K_{n}$ related to the next cluster to be created $C_{n}$. Add the first term from the list $V$ to the cluster $C_{n}$ and delete it from $V$.

3 ) (a) take the first node $i$ from the list $L$ that can be serviced by the vehicle assigned to the cluster $C_{n}$ and put it on the bottom of the list $K_{n}$. Initialize the cluster parameters $C_{n}$ :

$$
\begin{aligned}
& a C_{n} \leftarrow a_{i} \quad b C_{n} \leftarrow b_{i} \\
& w C_{n} \leftarrow w_{i} \quad \text { volC }_{n} \leftarrow \text { vol }_{i} \\
& \text { st }_{n} \leftarrow \mathrm{st}_{i}
\end{aligned}
$$

(b) Delete node $i$ from the $L$ list and make a copy of the current list $L$, and name it $L^{\prime}$.

4) Take the first node $j$ from the list $L^{\prime}$, and verify: (i) current cargo mass that should be delivered to the cluster $C_{n}$ plus $w_{j}$ does not exceed the mass cargo capacity $q_{v}$ of the assigned vehicle $v$; (ii) the current cargo volume that should be delivered to the cluster $C_{n}$ plus $v_{0} l_{j}$ does not exceed the volume cargo capacity of the assigned vehicle $v$; (iii) the node $j$ can be serviced by the vehicle $v$. If any of these three items is not completed, delete node $j$ from the list $L^{\prime}$ and repeat the procedure (4). Otherwise, proceed to step (5).

5) (a) Calculate the distance $d_{j i}$ between node $j$ and its closest node $i$ in the $K_{n}$ list. 
(b) Verify $d_{j i}$ to be less than the maximum allowed distance $d^{\text {max }}$. If it is not, delete node $j$ from the current list $L^{\prime}$ and turn back to the step (4). Otherwise, proceed to step (6).

6) Verify to satisfy the following constraint:

$$
a C_{n}+\mathrm{stC}_{n}+t_{i j}^{v}+\mathrm{st}_{j} \leq \max \left(b C_{n}, b_{j}\right)
$$

If it is not, delete node $j$ from the current list $L^{\prime}$ and turn back to the step (4). Otherwise, proceed to step (7).

7) Verify that the following constraint is satisfied:

$$
a C_{n}+\mathrm{stC}_{n}+t_{i j}^{v}+\Delta \geq a_{j}
$$

If it is not, close the cluster $C_{n}$ deleting the current list $L$ and recording the list $K_{n}$ which defines the cluster $C_{n}$, and turn back to the step (4). Otherwise, proceed to step (8).

8) (a) Put node $j$ at the bottom of the $K_{n}$ list and update the parameters of the cluster $C_{n}$ as follows:

$$
\begin{gathered}
w C_{n} \leftarrow w C_{n}+w_{j} \quad \operatorname{volC}_{n} \leftarrow \operatorname{vol}_{n}+\operatorname{vol}_{j} \\
\mathrm{st} C_{n} \leftarrow \max \left(\mathrm{stC}_{n}+t_{i j}^{v}+\mathrm{st}_{j}, a_{j}+\mathrm{st}_{j}-a_{i}\right)
\end{gathered}
$$

(b) If $b C_{n}<b_{j}$, then: $b C_{n} \leftarrow b_{j}$.

Otherwise, the latest finishing time of the service $b C_{n}$ is unchanged. Delete node $j$ from the lists $L$ and $L^{\prime}$ and proceed to the following step (9).

9) If the $L^{\prime}$ list is empty, keep the $K_{n}$ list that defines the cluster $C_{n}$ and proceed to the step (10). Otherwise, turn back to the step (4).

10) Repeat steps 2-9 until list $L$ is empty.

11) Calculate the time and road distance between any of two clusters defined by the algorithm. The distance between the two clusters can be calculated as the shortest distance between the two nodes belonging to those clusters, or as the average distance among all of their nodes, or like Max-Min distance.

The input into the procedure are the set of nodes (customers) $I$, the set of vehicles $V$, road and time distances among the nodes, serving time, the quantity of goods to be served and time windows. The aim of the procedure is to identify the set of feasible clusters that include the customers, where "the feasible cluster" means: (a) The load of the cluster can be assigned to a single vehicle; b) there is a route that connects the clusters and at the same time meets all the constraints of the time windows. The set of clusters that will be formed should be effective in the sense that (c) the vehicle waiting time due to early arrival has to be as short as possible and (d) an average distance per node inside the cluster for the assigned vehicle should remain low. In order to achieve these goals, the list of $L$ nodes is appropriately arranged in step (1) to enable the generation of the feasible low-cost clusters. Before adding the next node into the cluster being generated, its proximity with other nodes in the cluster is tested, as well as the time and capacity constraints (steps 4-7). The maximum resting time of $\Delta$ is allowed for the early arrival at a customer's location. If it is exceeded, integration of the node into the cluster is rejected (step 7).
In order to define the mathematical formulation using the term "cluster" and not the node, the terms "cluster time window" and "cluster service time" are introduced. The earliest time cluster $C_{n}$ servicing can start is given with $\min _{i \in C n}\left(a_{i}\right)$, while the latest time the servicing of $C_{n}\left(b C_{n}\right)$ has to be finished is updated by taking the maximum of $\left(b C_{n}, b_{i}\right)$, where $b C_{n}$ denotes the current time the cluster $C_{n}$ service has to be completed, and $b_{i}$ represents the appropriate value for the new input into the cluster $C_{n}$. Whenever a new node is added, the parameters of the cluster time window $\left[a C_{n}, b C_{n}\right]$ are updated. On the other hand, cluster $s t C_{n}$ service time is a good approximation of the total time the assigned vehicle spends visiting the cluster $C_{n}$. This time does not only include the service time of the assigned nodes, but also the travelling and resting time along the cluster.

As the fleet size is the variable in this problem, the procedure must choose the most efficient vehicles in order to complete a delivery process. This can be attained by sorting the vehicles, as is done in step (2) according to ascending value of cost-effectiveness. Finally, the time and road distance between any of the two clusters is determined in step (11). It should be noted that an arbitrary number of clusters may occur as a result of Phase 1 .

One vehicle is assigned for each of the clusters. As the number of clusters can be higher than the number of available vehicles, the vehicle list is expanded with an arbitrary number of virtual vehicles. These vehicles are only used for completing Phase 1. After the clusters are formed, virtual vehicles are removed from the list, while the available ones are left in it. Such reduced list is used as an input into Phase 2.

ii) Cluster-based multi-depot HFTW problem (Phase 2)

The aim of Phase 2 is to assign the clusters to the vehicles and to determine the order of those belonging to the same route by solving the compact version of MILP model, presented at the beginning of this section.

After completing Phase 2: (i) customers are assigned to the vehicles via the clusters; (ii) used vehicles are assigned to the depots; (iii) an almost optimal set of cluster-based routes is revealed; (iv) the order of cluster visiting belonging to the same route is obtained, which indirectly gives a partial arrangement of the order of customer visits serviced by the same vehicle.

\section{iii) Scheduling of one route (Phase 3)}

Scheduling the nodes inside the cluster and determining the starting time of the customer service for each of the routes is the aim of Phase 3. In order to achieve this, the VRPTW problem has to be formulated in the previous chapter as many times as there are routes in Phase 2. Including only the nodes from the clusters related to the same route, TSP formulation can be done for each of the routes. The relative order among clusters found in Phase 2 allows the further reduction of the number of variables of the order $S_{i j}$. 


\section{RESULTS DISCUSSION}

The proposed three-phase algorithm was tested on real data from one of the largest distribution companies in Bosnia and Herzegovina. The tested data that will be listed below are available at 4TU Research Data, research data centre [17], in order to be available to other scientists for their research and possible comparisons of the results. Because of the clustering mechanism and the division of the initial set of customers into the smaller regions, the proposed approach proved to be very efficient for bigger problems.

Results are listed below, presenting one instance of the problem for which it was necessary to find the optimal visits of 237 customers from one central depot, with an very heterogeneous fleet made of 16 vehicles. Vehicles and the depot had precisely defined working hours; customers had their appropriate time windows, including the additional constraints such as SDVRP (Site Dependent Vehicle Routing Problem), constraints where the customers, according to the ordered mass of goods, are serviced before because of a real cost reduction in this way.

In the initial phase, the proposed approach divided the input set of customers into 6 clusters, presented in different colours (except the red one) in Fig. 4. Geographically, they can be observed as separate units.

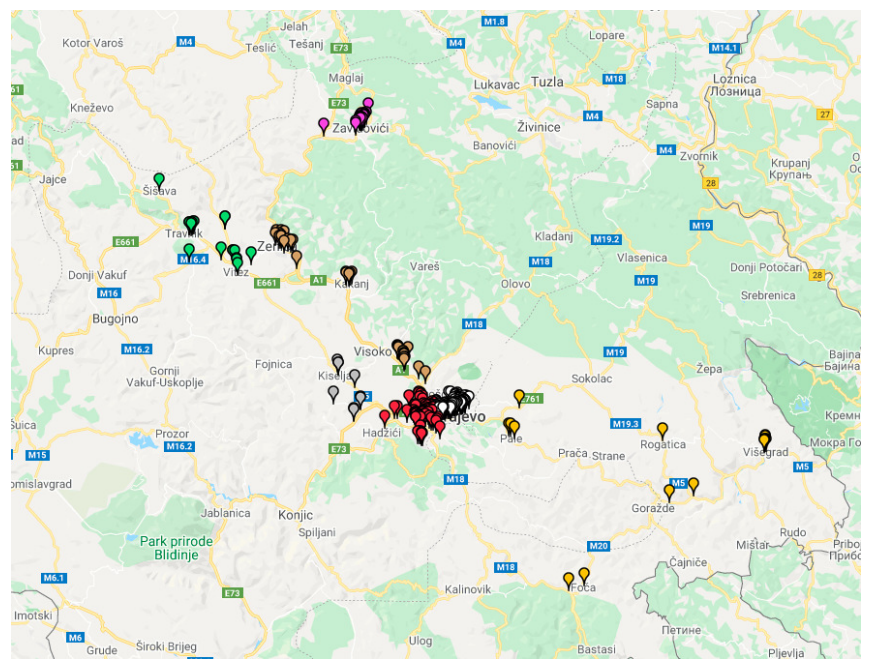

Fig. 4 Graphical presentation of cluster forming for the realistic example

Customers marked with red do not belong to any of the clusters; they are located near the given depot, and they are potential candidates for completing the routes related to the previously defined 6 clusters. It is performed in Phase 2 of the proposed approach, while in Phase 3 the optimal order within each of the created clusters is determined.

Figure 5 shows the obtained transport route for one vehicle combining the cluster presented in yellow in Fig. 4 (customers from 1 to 15 in Fig. 5), with the customers gathered around the depot, not initially belonging to any of the clusters (customers from 16 to 24 in Fig. 5).

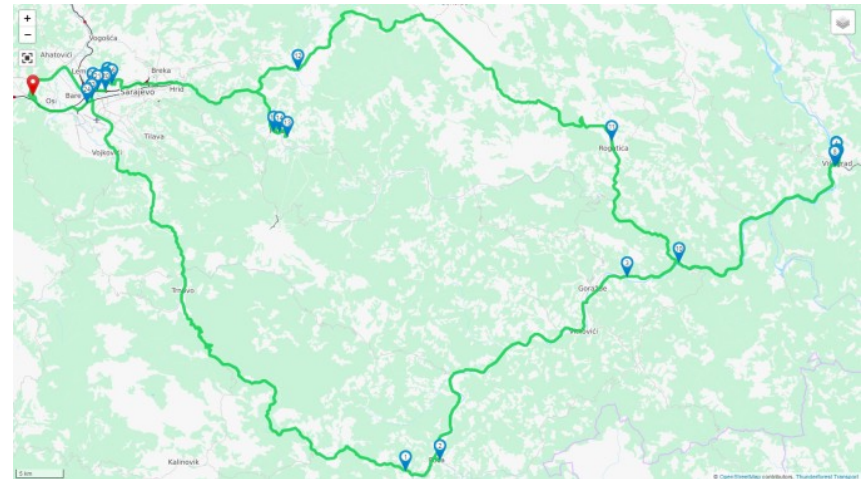

Fig. 5 A transportation route for the vehicle: combination of more clusters

Order of customer visits for the mentioned testing example presented in Table I.

TABLE I - TESTING RESULTS ON REALISTIC COMPANY DATA: ORDER OF CUSTOMER VISITS

\begin{tabular}{|c|c|}
\hline Vehicle & $\begin{array}{l}\text { Customers visits order (customers are marked with } \\
\text { their corresponding codes) } \\
\text { * The code } 1000 \text { indicates a depot, which means that } \\
\text { each route begins and ends at the depot }\end{array}$ \\
\hline A08-J-523 & $\begin{array}{l}1000,140513,923425,5231,4763,921850,5992,5383,923487, \\
920355,5274,142963,923509,923482,923414,5382,941071, \\
144566,139746,141055,142571,5172,5934,920263,923781 \\
923687,5029,143352,137557,923002,1000\end{array}$ \\
\hline A09-J-051 & $\begin{array}{l}1000,131821,940895,4302,5971,139518,941079,922465 \\
923485,941068,133081,139095,15297,135665,923504, \\
921685,135952,134436,134757,941076,133857,922629, \\
923474,142730,922545,5281,5711,133301,4421,15194,1000\end{array}$ \\
\hline E03-O-502 & $\begin{array}{l}1000,133584,131380,923857,921044,140232,921804,923460 \\
140231,924891,137608,923453,132979,131423,5599,4334, \\
15266,7624,131422,1000\end{array}$ \\
\hline E57-A-655 & $\begin{array}{l}1000,923508,5380,922377,135790,133202,5270,5716,15077 \\
139770,5387,923491,144832,5272,5384,137825,921026, \\
1000\end{array}$ \\
\hline E81-M-660 & $\begin{array}{l}1000,923704,139623,920779,5922,141457,15319,5379 \\
135146,140851,140594,941290,1000\end{array}$ \\
\hline E85-T-014 & $\begin{array}{l}1000,138152,138320,924238,133238,132293,924186,134190 \\
137739,144596,136433,138508,139038,923370,15082, \\
143746,143101,5285,923502,15074,144879,136655,133672, \\
921045,920279,1000\end{array}$ \\
\hline E90-J-092 & $\begin{array}{l}1000,924298,7699,5984,924542,144765,923479,923364 \\
924442,923498,923490,5824,921334,5712,1000\end{array}$ \\
\hline J37-M-937 & $\begin{array}{l}1000,15146,144419,5710,139499,5999,140671,143456 \\
134865,142725,133927,941289,921902,921438,923287 \\
920952,138999,143622,138468,140937,133984,143422 \\
920264,1000\end{array}$ \\
\hline $\mathrm{J} 67-\mathrm{T}-730$ & $\begin{array}{l}1000,4431,5908,134972,15043,143689,133561,4981,921039, \\
924531,136232,142935,136638,923488,922775,4344,923511, \\
941096,923493,941092,140079,1000\end{array}$ \\
\hline M04-A-376 & $\begin{array}{l}1000,137617,131450,920108,15155,921541,920152,139039 \\
137177,5113,5512,922425,139564,133867,10220,15154 \\
143513,924012,1000\end{array}$ \\
\hline M80-T-354 & $1000,920604,923286,923514,923771,923521,1000$ \\
\hline O40-K-191 & $1000,924594,133725,1000$ \\
\hline O40-K-192 & $1000,920286,1000$ \\
\hline O78-K-073 & $\begin{array}{l}1000,941319,922313,5436,923483,5277,941069,923471 \\
138126,5264,923884,15287,138138,139378,921261,1000\end{array}$ \\
\hline T34-E-701 & $\begin{array}{l}1000,15133,5164,922607,940840,921579,143442,131671, \\
923495,143680,5434,5822,923500,923480,138219,131636, \\
141110,1000\end{array}$ \\
\hline
\end{tabular}

Customers' arrival times for the routes presented in Table I are respectively presented in Table II. Time windows of each of the customers are shown in brackets. First and last times in Table II show the departure time of the vehicles from the depot, as well as the return period of the vehicle to the depot. 
TABLE II - TESTING RESULTS ON REALISTIC COMPANY DATA: ORDER OF CUSTOMER VISITS

\begin{tabular}{cc} 
Customers visits times \\
$\begin{array}{c}\text { Vehicle } \\
\text { (distance }[\mathrm{km}])\end{array}$ & $* \begin{array}{c}\text { Each route starts and ends at the depot }- \text { the } \\
\text { first and last time for each vehicle }\end{array}$ \\
\hline
\end{tabular}

07:44, 08:01-08:15 (- 19:01), 08:19-08:34 (-19:01), 08:3708:52 ( - 19:01), 08:55-09:16 (- 19:01), 09:17-09:33 (19:01), 09:34-10:00 (08:00 - 17:00), 10:01-10:21 (08:00 17:00), 10:21-10:41 ( - 19:01), 10:43-11:01 ( - 19:01), 11:0211:18 (08:00 - 17:00), 11:18-11:35 ( - 19:01), 11:36-11:54 ( $19: 01), 11: 56-12: 13(-19: 01), 12: 13-12: 34(-19: 01), 12: 35-$

A08-J-523

$(114.802 \mathrm{~km})$ 12:50 (08:00 - 17:00), 12:56-13:12 ( - 19:01), 13:13-13:29 ( 19:01), 13:33-13:53 (- 19:01), 14:03-14:18 (-19:01), 14:20$14: 36(-19: 01), 14: 50-15: 06(-19: 01), 15: 13-15: 32(08: 00-$ 17:00), 15:33-15:50 (08:00 - 18:00), 15:50-16:07 ( - 19:01), 16:17-16:33 (- 19:01), 16:41-16:56 ( - 19:01), 16:56-17:11 ( $19: 01), 17: 15-17: 30$ ( - 19:01), 17:37-17:52 (- 19:01), 18:19 07:41, 08:00-08:36 (08:00 - 17:00), 08:37-08:52 ( - 19:01), 08:56-09:14 ( - 19:01), 09:16-09:38 (08:00 - 17:00), 09:39 09:55 (08:00 - 19:00), 09:56-10:11 ( - 19:01), 10:11-10:26 ( 19:01), 10:26-10:41 ( - 19:01), 10:42-11:01 ( - 19:01), 11:05$11: 21(08: 00-19: 00), 11: 21-11: 36(-19: 01), 11: 40-11: 55$ (08:00 - 17:00), 11:58-12:14 ( - 19:01), 12:14-12:30 ( -

A09-J-051 $(53.249 \mathrm{~km})$ $13: 05(-19: 01)$ 13:06-13:22 ( - 19:01), 13:22-13:38 (08:00 - 17:00), 13:4013:55 ( - 19:01), 13:57-14:13 ( - 19:01), 14:14-14:42 ( 19:01), 14:42-15:03 ( - 19:01), 15:04-15:20 (08:00 - 17:00), $15: 24-15: 38$ ( - 19:01), 15:39-15:57 (08:00 - 17:00), 16:00$16: 15(08: 00$ - 17:00), 16:16-16:41 (08:00 - 18:00), 16:43$16: 59$ ( - 19:01), 17:00-17:14 (08:00 - 19:00), 17:32 06:55, 08:01-08:18 ( - 19:01), 08:20-08:35 (08:00 - 17:00), 08:36-08:51 ( - 19:01), 09:11-09:25 ( - 19:01), 09:40-09:55 ( 19:01), 09:56-10:10 ( - 19:01), 10:13-10:28 ( - 19:01), 10:29-

$\begin{array}{ll}\text { E03-O-502 } & 10: 44(-19: 01), 11: 12-11: 35(-19: 01), 12: 04-12: 19(- \\ (265.176 \mathrm{~km}) & 19: 01), 12: 19-12: 34(-19: 01), 12: 50-13: 06(-19: 01), 13: 06-\end{array}$ 13:23 (08:00 - 17:00), 13:33-13:50 ( - 19:01), 13:54-14:12 ( 19:01), 14:12-14:29 (08:00 - 17:00), 14:30-14:49 ( - 19:01), $14: 55-15: 10(08: 00-17: 00), 16: 15$

07:53, 08:00-08:17 ( - 19:01), 08:29-08:45 (08:00 - 17:00), 08:45-09:01 (08:00 - 17:00), 09:03-09:20 ( - 19:01), 09:2809:59 (08:00 - 18:00), 10:03-10:19 (08:00 - 17:00), 10:20-

E57-A-655 $(44.618 \mathrm{~km})$ 10:35 (08:00 - 17:00), 10:38-11:04 (08:00 - 17:00), 11:0811:32 ( - 19:01), 11:47-12:04 (08:00 - 17:00), 12:05-12:23 ( 19:01), 12:23-12:44 (08:00 - 18:00), 12:46-13:04 (08:00 17:00), 13:04-13:22 (08:00 - 17:00), 13:23-13:50 ( - 19:01), 13:55-14:15 (08:00 - 18:00), 14:27

07:49, 08:00-08:19 ( - 19:01), 08:27-08:44 ( - 19:01), 08:44 09:07 (08:00 - 18:00), 09:07-09:37 (08:00 - 17:00), 09:40

$\begin{array}{ll}\text { E81-M-660 } & 10: 15(08: 00-18: 00), 10: 16-10: 40(08: 00-17: 00), 10: 41- \\ (31.868 \mathrm{~km}) & 11: 02(07: 00-18: 00), 11: 04-11: 21(-19: 01), 11: 22-11: 38\end{array}$ $\begin{array}{ll}(31.868 \mathrm{~km}) & 11: 02(07: 00-18: 00), 11: 04-11: 21(-19: 01), 11: 22-11: 38 \\ & (08: 00-17: 00), 11: 41-11: 58(08: 00-18: 00), 12: 00-12: 17(-\end{array}$ 19:01), 12:29

06:38, 08:01-08:16 ( - 19:01), 08:21-08:36 ( - 19:01), 09:1709:32 ( - 19:01), 10:06-10:21 (- 19:01), 10:21-10:36 ( 19:01), 10:38-10:59 ( - 19:01), 11:00-11:20 ( - 19:01), 11:2011:39 (08:00 - 18:00), 11:39-12:08 (08:00 - 18:00), 12:37$12: 52$ ( - 19:01), 13:11-13:26 ( - 19:01), 14:15-14:32 (-

E85-T-014

$(301.048 \mathrm{~km})$ 19:01), 14:47-15:02 ( - 19:01), 15:06-15:27 (08:00 - 17:00), 15:29-15:45 ( - 19:01), 16:11-16:26 ( - 19:01), 16:28-16:47 (08:00 - 17:00), 16:48-17:04 ( - 19:01), 17:04-17:24 ( 19:01), 17:24-17:44 (08:00 - 18:00), 17:46-18:01 ( - 19:01), 18:02-18:18 ( - 19:01), 18:20-18:36 ( - 19:01), 18:37-18:52 ( 19:01), 19:01

07:03, 08:01-08:51 ( - 19:01), 08:52-09:13 ( - 19:01), 09:2010:10 (08:00 - 18:00), 10:15-10:31 ( - 19:01), 10:33-10:50 ( -

E90-J-092

$(163.846 \mathrm{~km})$ 19:01), 10:52-11:07 ( - 19:01), 11:10-12:00 ( - 19:01), 12:0212:18 ( - 19:01), 12:20-12:35 ( - 19:01), 12:39-12:54 ( 19:01), 12:55-13:34 (08:00 - 17:00), 13:38-13:53 (08:00 17:00), 14:03-14:22 (08:00 - 17:00), 15:16 07:45, 08:00-08:15 ( - 19:01), 08:16-08:38 (08:00 - 17:00), 08:39-08:55 (08:00 - 17:00), 08:55-09:11 ( - 19:01), 09:1209:31 (08:00 - 17:00), 09:32-09:47 ( - 19:01), 09:47-10:03 (08:00 - 18:00), 10:05-10:21 ( - 19:01), 10:21-10:37 ( -

J37-M-937 19:01), 10:37-10:55 (- 19:01), 11:00-11:15 ( - 19:01), 11:16$(41.36 \mathrm{~km}) \quad 11: 32(-19: 01), 11: 32-11: 47(-19: 01), 11: 48-12: 04(-$ 19:01), 12:06-12:27 (08:00 - 17:00), 12:28-12:45 ( - 19:01), 12:46-13:22 (08:00 - 17:00), 13:23-13:41 (08:00 - 17:00), 13:42-13:57 ( - 19:01), 14:00-14:15 ( - 19:01), 14:16-14:32 (08:00 - 18:00), 14:35-15:25 (08:00 - 18:00), 15:40
$(113.851 \mathrm{~km})$

09:42 (08:00 - 17:00), 09:43-10:00 (08:00 - 18:00), 10:0110:16 (08:00 - 17:00), 10:34-10:50 ( - 19:01), 10:50-11:05 ( 19:01), 11:06-11:20 (08:00 - 17:00), 11:21-11:37 ( - 19:01), 11:42-11:57 ( - 19:01), 11:59-12:27 (08:00 - 18:00), 12:27 12:44 ( - 19:01), 12:45-13:07 ( - 19:01), 13:07-13:27 ( 19:01), 13:29-13:45 ( - 19:01), 13:46-14:05 ( - 19:01), 14:0514:24 ( - 19:01), 14:25-14:44 ( - 19:01), 14:49-15:09 ( 19:01), 15:16-15:31 ( - 19:01), 15:51

06:30, 08:15-08:31 ( - 19:01), 08:31-08:53 ( - 19:01), 08:5509:11 (- 19:01), 09:11-09:28 (- 19:01), 09:28-09:44 ( 19:01), 09:44-10:05 ( - 19:01), 10:05-10:20 ( - 19:01), 10:20-

M04-A-376 $10: 36(-19: 01), 10: 37-11: 00(-19: 01), 11: 02-11: 16(-$ 19:01), 11:18-11:34 ( - 19:01), 11:35-12:02 (08:00 - 17:00), 12:09-12:24 ( - 19:01), 12:33-12:50 ( - 19:01), 12:53-13:10 ( 19:01), 13:14-13:36 ( - 19:01), 13:38-13:55 ( - 19:01), 15:41 M80-T-354 07:40, 08:00-08:17 ( - 19:01), 08:18-08:34 ( - 19:01), 08:35\begin{tabular}{ll} 
(33.444 km) & $08: 50(-19: 01), 08: 50-09: 20(-19: 01), 09: 23-10: 23(-$ \\
\hline & $19: 01), 10: 39$
\end{tabular}

O40-K-191

$(243.667 \mathrm{~km})$

O40-K-192

(19.283 km)

06:30, 08:18-08:41 ( - 19:01), 08:56-09:13 ( - 19:01), 10:46

07:47, 08:00-08:50 (08:00 - 18:00), 09:01

07:39, 08:00-08:18 ( - 19:01), 08:19-08:34 ( - 19:01), 08:3509:07 (08:00 - 19:00), 09:08-09:26 ( - 19:01), 09:26-09:41

O78-K-073 (08:00 - 17:00), 09:41-10:00 ( - 19:01), 10:00-10:23 ( -

(42.028 km) 19:01), 10:25-10:47 (-19:01), 10:48-11:11 (08:00 - 17:00), 11:12-11:31 ( - 19:01), 11:32-11:51 (08:00 - 17:00), 11:5112:08 ( - 19:01), 12:18-12:32 ( - 19:01), 12:34-13:15 (08:00 18:00), 13:31

07:51, 08:00-08:31 (08:00 - 18:00), 10:22-10:38 ( - 19:01), 12:15-12:30 ( - 19:01), 12:31-12:49 ( - 19:01), 12:50-13:07 ( 19:01), 13:08-13:24 ( - 19:01), 13:25-13:42 (08:00 - 17:00),

T34-E-701 13:43-14:04 ( - 19:01), 14:05-14:34 (08:00 - 18:00), 14:37 14:58 (07:00 - 18:00), 15:01-15:16 (08:00 - 17:00), 15:1915:35 ( - 19:01), 15:37-15:58 ( - 19:01), 16:00-16:16 (08:00 17:00), 16:18-16:39 (08:00 - 17:00), 16:39-16:55 (08:00 17:00), 17:03

Table II shows the total number of kilometres for the used testing instance of the realistic routing for each of the vehicles from the fleet.

The proposed approach managed to find the optimal solution by using 15 vehicles from a fleet of 16 available, thus satisfying all the set constraints. During a testing period of more than a month, using the proposed approach, compared to the previous routing based on long term experience of the transport managers, produced savings from the possibility to use one or two vehicles less during the daily routing. From the financial aspect of the company, those savings range from 10 to $20 \%$, which is really more than satisfactory in real-time environments.

From the previous results, it can be concluded that the algorithm was always able to find solutions that completely fit the constraints. Therefore, costs were cut due to the more efficient use of the fleet, particularly the use of smaller vehicles. Larger vehicles, which have greater costs, were only used when the smaller vehicles could not serve all the customers; meaning that the use of larger vehicles was absolutely necessary. In these scenarios, the algorithm routed the larger vehicles to serve as few customers as possible and those closer to the depot, consequently minimizing the cost of these vehicles.

Looking at the algorithm's run-time, it is the number of customers and their constraints that have the greatest impact. Aside from that, the peculiarities of a fleet of vehicles, which include its size and type vehicles available, also have an effect on the run-time. 


\section{Conclusion}

The optimization of the transport system, from the perspective of the logistical approach of transport management, is primarily achieved by the optimization of the transport flows of goods and using the transport means. When it comes to the use of transport means, the optimization process solves the problems based on the basic characteristics of the routing problem. The problem of determining the optimal route (path) of the transport means performing the service on the transport network, in terms of minimizing the distance, travel time or service costs (transport).

In theory and practice, there are numerous models for solving these and similar problems. Most of the efficient models for solving complex VPR problems are based on a heuristic approach founded on clustering techniques. An innovative multiphase, cluster-based model was presented in this work. The model presented is able to successfully solve VRP problems of large dimensions, satisfying the realistic constraints such as the heterogenic vehicle fleet, time windows of the customers, constraints of what customer can be served by a specific vehicle, etc. From the aspect of practical application, this approach generated significant results on the examples of the transport route optimization of one of the biggest distribution companies in Bosnia and Herzegovina.

The presented approach can be additionally improved by extending it for the multi-depot transport option, as well as including additional constraints such as multiple trips, possibility of returning goods during the one transport route, etc. Also, in the initial phase of the cluster creation, heuristic approaches such as ant colonies, swarm of bees or heuristic neighbors with a changing environment can be applied.

\section{REFERENCES}

[1] Dantzig, G. B., and Ramser, J. H. (1959). The truck dispatching problem. Management science, Vol. 6(1), 80-91. doi: 10.1287/ mnsc. 6.1 .80

[2] Hall, R. (2006). On the road to integration. OR/MS Today 33(3), $50-57$.

[3] Grosso, R., Munuzuri, J., Escudero-Santana, A., and Barbadilla-Martín, E. (2018). Mathematical Formulation and Comparison of Solution Approaches for the Vehicle Routing Problem with Access Time Windows. Complexity. doi:10.1155/2018/4621694

[4] Nalepa, J., and Blocho, M. (2016). Adaptive memetic algorithm for minimizing distance in the vehicle routing problem with time windows. Soft Computing, Vol. 20 (6), 2309-2327. doi: 10.1007/ s00500-015-1642-4
[5] Dixit, A., Mishra, A., and Shukla, A. (2019). Vehicle Routing Problem with Time Windows Using Meta-Heuristic Algorithms: A Survey. In: Yadav N., Yadav A., Bansal J., Deep K., Kim J. (eds) Harmony Search and Nature Inspired Optimization Algorithms. Advances in Intelligent Systems and Computing, Vol. 741, 539-546. doi: 10.1007/978-981-13-0761-4_52

[6] Goel, R., and Maini, R. (2018). A hybrid of ant colony and firefly algorithms (HAFA) for solving vehicle routing problems. Journal of Computational Science, Vol. 25, 28-37. doi: 10.1016/ j.jocs.2017.12.012

[7] Mahmudy, W. F. (2014). Improved Simulated Annealing for Optimization of Vehicle Routing Problem With Time Windows (VRPTW). Kursor, Vol. 7(3). doi: 10.21107/KURSOR.V7I3.1092

[8] Osaba, E., Yang, X. S., and Del Ser, J. (2020). Is the Vehicle Routing Problem Dead? An Overview Through Bioinspired Perspective and a Prospect of Opportunities. In: Yang XS., Zhao YX. (eds) NatureInspired Computation in Navigation and Routing Problems. Springer Tracts in Nature-Inspired Computing. Springer, Singapore, doi: 10.1007/978-981-15-1842-3 3

[9] Dondo, R., and Cerdá, J. (2007). A cluster-based optimization approach for the multi-depot heterogeneous fleet vehicle routing problem with time windows. European Journal of Operational Research, Vol. 176(3), 1478-1507. doi: 10.1016/j.ejor.2004.07.077

[10] Žunić, E., Đonko, D., and Buza., E. (2020). An adaptive data-driven approach to solve real-world vehicle routing problems in logistics. Complexity. doi: 10.1155/2020/7386701

[11] Žunić, E., Hindija, H., Beširević, A., Hodžić, K., and Delalić, S. (2018). Improving Performance of Vehicle Routing Algorithms using GPS Data. 14th Symposium on Neural Networks and Applications (NEUREL), Belgrade, Serbia, 2018, 1-4. doi: 10.1109/ NEUREL.2018.8586982

[12] Žunić, E., Djedović, A., and Đonko, D. (2017). Cluster-based analysis and time-series prediction model for reducing the number of traffic accidents. International Symposium ELMAR, 25-29. doi: 10.23919/ ELMAR.2017.8124427

[13] Žunić, E., and Đonko, D. (2019). Parameter setting problem in the case of practical vehicle routing problems with realistic constraints. 2019 Federated Conference on Computer Science and Information Systems (FedCSIS), 755-759. doi: 10.15439/2019F 194

[14] Žunić, E., Delalić, S., Hodžić, K., Beširević, A., and Hindija, H. (2018). Smart warehouse management system concept with implementation. 14th Symposium on Neural Networks and Applications (NEUREL), 1-5. doi: 10.1109/NEUREL.2018.8587004

[15] Zunic, E., Besirevic, A., Delalic, S., Hodzic, K., and Hasic, H. (2018). A generic approach for order picking optimization process in different warehouse layouts. in 2018 41st International Convention on Information and Communication Technology, Electronics and Microelectronics (MIPRO). doi: 10.23919/MIPRO.2018.8400183

[16] Žunić, E., Djedović, A., and Đonko, D. (2016). Application of Big Data and text mining methods and technologies in modern business analyzing social networks data about traffic tracking. XI International Symposium on Telecommunications (BIHTEL), 1-6. doi: 10.1109/ BIHTEL.2016.7775717

[17] Žunić, E. (Emir). (2018). Real-world VRP benchmark data with realistic non-standard constraints - input data and results. 4TU.Centre for Research Data. Dataset. https://doi.org/10.4121/uuid:598b19d1df64-493e-991a-d8d655dac3ea 